\title{
Evidence for Thermal Decomposition Contributions to the Mass Spectra of Chlorinated Phenoxyacid Herbicides Obtained by Particle Beam Liquid Chromatography Mass Spectrometry
}

\author{
L. Donnelly Betowski \\ U.S. Environmental Protection Agency, Las Vegas, Nevada, USA \\ Chris M. Pace and Mark R. Roby \\ Lockheed Engineering and Sciences Co., Las Vegas, Nevada, USA
}

\begin{abstract}
The spectral quality of a group of chlorinated phenoxyacid herbicides has been shown to degrade under certain conditions upon introduction into the mass spectrometer by a particle beam interface. Experiments were performed to investigate these changes in spectra. Normalized ion chromatograms were generated for the herbicides, and the results showed a broadening of the profiles of some ions, indicating a longer residence time in the ion source. These ions were postulated as coming from the ionization of thermal degradation products from the herbicides. The generation of these ions was dependent on ion source temperature, analyte concentration, and, by implication, ion source cleanliness. Tandem mass spectrometry experiments were performed on these ions from the herbicides and ions from the corresponding phenols. The tandem mass spectra of the ions from the herbicides were similar to the tandem mass spectra of the ions from the phenols. Therefore, it appears that the particle beam mass spectra of the chlorinated phenoxyacid herbicides are composite spectra with contributions from the gas phase ionization of the parent herbicides and thermal decomposition products. (J Am Soc Mass Spectrom 1992, 3, 823-830)
\end{abstract}

$\mathrm{P}$ article beam (PB) high performance liquid chromatography/mass spectrometry (HPLC/MS) is a relatively new technique adapted from the monodisperse aerosol generation interface for combined LC/MS technology developed by Willoughby and Browner [1]. The advantage of using the $\mathrm{PB}$ interface for LC/MS over some of the other interfaces (e.g., thermospray and electrospray) lies mainly in the type of spectra generated. Once most of the mobile phase has been removed by a two-stage momentum separator, the analyte particles enter the mass spectrometer ion source at a low pressure, where they are vaporized on impact with ion source surfaces and subsequently ionized by $70-\mathrm{eV}$ (nominal) electrons. The electron ionization (EI) mass spectra generally contain substantial structural information. It is the EI mass spectral capa-

Address reprint requests to L. Donnelly Betowski, U.S. EPA. Environmental Monitoring Systems Laboratory, Post Office Box 93478, Las Vegas, NV 89193-3478. bility of the $\mathrm{PB}$ interface that has captured the interest of many environmental chemists.

The development of LC/MS interfaces has been driven by the inadequacies of gas chromatography (GC), especially with respect to polar, nonvolatile, and thermally labile compounds. Although LC systems alone are suitable for these types of compounds, conventional LC detectors do not provide the analyte identification capabilities of the mass spectrometer.

Phenoxyacid herbicides are compounds that have presented difficulties in GC analyses. Analytical methodology for these compounds has relied on the use of derivatization steps prior to GC with electron capture detection [2-5]. In an attempt to avoid the derivatization step, researchers have recently applied LC/MS methods to the analysis for phenoxyacid herbicides. Direct liquid introduction $[6,7]$, thermospray [8-12], and PB [13-16] interfaces have all been used in the identification and quantification of these compounds. During a project to develop an analytical method for the determination of phenoxyacid herbicides by particle beam LC/MS, we encountered sub- 
stantial difficulties with poor method precision and accuracy. Part of these difficulties were attributed to variations in the particle beam mass spectra of phenoxyacid herbicides. This paper presents a look at these compounds by PB LC/MS with emphasis on thermal degradation that may be occurring in the $\mathrm{PB}$ ion source.

\section{Experimental}

Most of the experiments were performed on a Hewlett-Packard (HP) 5988A mass spectrometer (Avondale, PA) with an HP 1090L LC and an HP 59980A PB interface. The mass spectrometer was controlled by an HP 59970A MS Chem Station.

The LC flow rate was $0.25 \mathrm{~mL} / \mathrm{min}$ unless otherwise specified. A Spherisorb S3 C-8 column (3- $\mu \mathrm{m}$ particle diameter, 2-mm i.d. $\times 100 \mathrm{~mm}$, Phase Separations, Norwalk, CT) was used for separation. A $1 \%$ acetic acid solution in the mobile phase was found sufficient to suppress dissociation of the acid herbicides.

Routine PB parameters were as follows: nebulizer setting 12 , nebulization helium pressure 30 to $50 \mathrm{psi}$, desolvation chamber temperature 45 to $55^{\circ} \mathrm{C}$, and $\mathrm{PB}$ probe distance to the source $0.5 \mathrm{~mm}$. The $\mathrm{PB}$ desolvation chamber vacuum pressure was estimated at 200 torr by the instrument manufacturer. The pressure in the first stage of the momentum scparator was typically 10 torr, and that of the second stage was 0.5 torr as measured by Hastings-Raydist gauges. The mass spectrometer ion source for these studies was slightly modified. First, a stainless steel plug was inserted into the GC inlet of the source. Second, the PB inlet to the source was drilled out to a larger diameler by the instrument manufacturer. An ion source temperature of $250{ }^{\circ} \mathrm{C}$ was used throughout unless otherwise specified. A typical mass spectrometer operating pressure of $1.2 \times 10^{-5}$ torr was measured by a Bayard-Alpert ion gauge tube.

The tandem mass spectrometry (MS/MS) experiments were performed with a Finnigan MAT (San Jose, CA) TSQ45 triple quadrupole mass spectrometer. A collision energy of $30 \mathrm{eV}$ was used for the collisionally activated dissociation (CAD) daughter ion experiments at pressures of 0.2 to 1 mtorr; parent ion experiments were performed at a collision energy of $20 \mathrm{eV}$ and approximately 1mtorr. An Extrel (Millipore/Extrel, Pittsburgh, PA) ThermaBeam LC/MS interface was adapted for use with the TSO45 triple quadrupole mass spectrometer, and compounds were injected directly into the inferface without an analytical column with $100 \%$ methanol at a flow rate of $0.3 \mathrm{~mL} / \mathrm{min}$. The nebulization helium pressure was 35 psi; the ion source temperature was approximately $190{ }^{\circ} \mathrm{C}$. A J \& W DB-5 fused silica capillary column (Folsom, CA) was used for the GC/MS/MS experiments.

The acid herbicide and phenol standards (>97\% purity) were obtained from the U.S. Environmental Protection Agency Repository (Research Triangle Park,
NC). Standard solutions were prepared with acetonitrile (Burdick and Jackson, Muskegon, MI).

\section{Results and Discussion}

Variation in the particle beam mass spectra of two phenoxyacid herbicides, 2-methyl-4-chlorophenoxyacetic acid (MCPA) and 2,4-dichlorophenoxyacetic
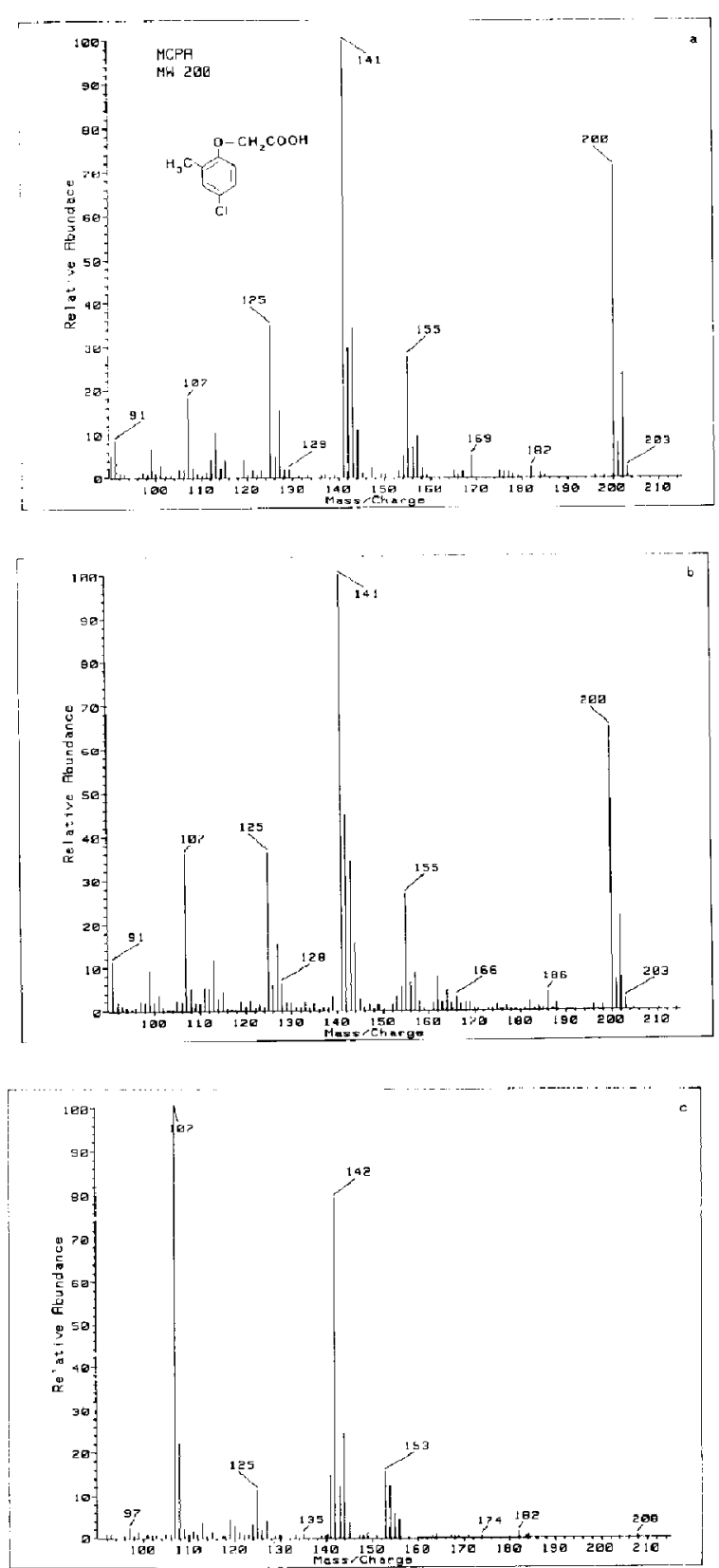

Figure 1. Comparative mass spectra of MCPA. (a) DIP mass spertrum of MCPA. (b) Particle beam mass spectrum of MCPA that is library matchable. (c) Particle beam mass spectrum of MCPA that is not library matchable. 

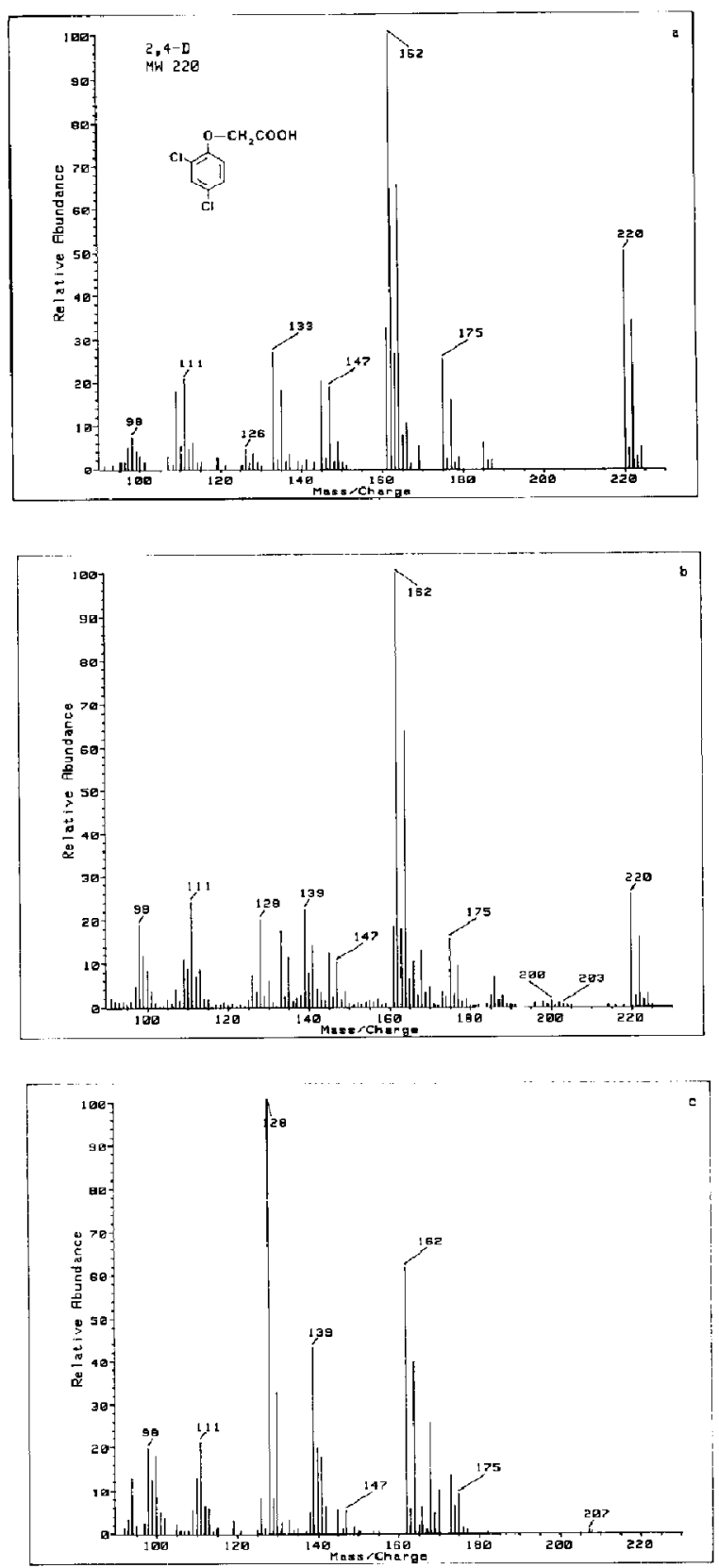

Figure 2. Comparative mass spectra of 2,4-D. (a) DIP mass spectrum of 2,4-D. (b) Particle beam mass spectrum of 2,4-D that is library matchable. (c) Particle beam mass spectrum of 2,4-D that is not library matchable.

acid (2,4-D), is illustrated in Figures 1 and 2, respectively, along with the corresponding direct insertion probe (DIP) mass spectra. Figures $1 \mathrm{a}$ and $2 \mathrm{a}$ are the DIP mass spectra acquired on the Hewlett-Packard instrument at an ion source temperature of $250^{\circ} \mathrm{C}$ with $2.5 \mu \mathrm{g}$ of compound applied to the probe. Figures $1 \mathrm{~b}$ and $2 \mathrm{~b}$ show the particle beam mass spectra of MCPA and 2,4-D, respectively, from an on-column injection of a standard solution containing eight phenoxyacid herbicides at $200 \mu \mathrm{g} / \mathrm{mL}$ each and an ion source temperature of $250{ }^{\circ} \mathrm{C}$. Specifically, $10 \mu \mathrm{L}$ of the standard solution was injected onto a C-8 reversed phase column eluted with a methanol/1\% acetic acid, $0.01 \mathrm{M}$ ammonium acetate gradient at a flow rate of 0.25 $\mathrm{mL} / \mathrm{min}$. The initial solvent composition (25\% methanol) was held for $2 \mathrm{~min}$; the methanol content was then increased linearly to reach $60 \%$ at $25 \mathrm{~min}$.

Figures $1 \mathrm{c}$ and $2 \mathrm{c}$ also show particle beam mass spectra of MCPA and 2,4-D, respectively, acquired several weeks prior to spectra $b$ and under different chromatographic conditions. In this case, $10 \mu \mathrm{L}$ of a $100 \mu \mathrm{g} / \mathrm{mL}$ standard solution was injected onto a C-18 reversed phase column (Supelcosil LC-18, 5- $\mu \mathrm{m}$ particle diameter, $2.1 \mathrm{~mm} \times 250 \mathrm{~mm}$, Supelco, Bellefonte, PA) eluted with a methanol/1\% acetic acid gradient but without ammonium acetate and at a flow rate of $0.4 \mathrm{~mL} / \mathrm{min}$. The initial mobile phase consisted of $50 \%$ methanol (containing $1 \%$ acetic acid) and $50 \%$ water (containing 1\% acetic acid). This composition was held for $2 \mathrm{~min}$; the methanol content was increased linearly to reach $60 \%$ over a period of $10 \mathrm{~min}$ and then to $100 \%$ over the next $6 \mathrm{~min}$. The ion source temperature was $250{ }^{\circ} \mathrm{C}$. Besides differences in the chromatographic conditions and concentration, spectra $b$ were acquired with an ion source that had just been cleaned whereas spectra $c$ were acquired with an ion source that had been in use several weeks since last cleaned.

The DIP spectra (Figures 1a and 2a) match reference spectra found in the Wiley Library [17] provided with the instrument operating system and are also consistent with the mass spectra reported in the literature for MCPA and 2,4-D [18]. The particle beam $b$ spectra are also library matchable and are similar to the corresponding DIP spectra. Comparison of the particle beam b spectra with the DIP spectra shows some minor differences. The MCPA particle beam mass spectrum 1b shows an increased relative abundance of ions at $m / z 107$ and 142. The 2,4-D particle beam mass spectrum $2 \mathrm{~b}$ shows an increased relative abundance of ions at $m / z 128$ and 139 and decreased relative abundance of the molecular ion at $m / z 220$.

Comparison of the particle beam mass spectra $c$ with spectra $a$ and $b$ shows major differences. The MCPA particle beam mass spectrum 1c lacks the molecular ion at $m / z 200$, the ion at $m / z 107$ has become the base peak, and the $m / z$ 141:142 ratio has changed from about $2: 1$ in spectrum $1 \mathrm{~b}$ to about 1:4 in spectrum 1c. The 2,4-D particle beam mass spectrum 2 c lacks the molecular ion at $m / z 220$, the ion at $m / z$ 128 has become the base peak, and the relative abundance of ions at $m / z 139$ and 168 has increased.

The mass spectra in Figures 1 and 2 represent the extremes of our abservations. The extent of these variations cannot be attributed solely to differences in instrument tuning. More frequently, we observe particle beam mass spectra of MCPA and 2,4-D that are intermediate to the extremes. We have also observed particle beam mass spectral variation with other phe- 
noxyacid herbicides, namely, 2,4,5-T and Silvex. Data received from other laboratories during an interlaboratory study on the application of LC/MS to the analysis of phenoxyacid herbicides also exhibit considerable mass spectral variation using particle beam type LC/MS instruments [19]. Variation in the particle beam mass spectra of phenoxyacid herbicides appears to be characteristic for this class of compounds. Other compound classes that we have examined by particle beam LC/MS-for example, esters of phenoxyacid herbicides and polycyclic aromatic hydrocarbons-do not exhibit the variable mass spectra observed for the phenoxyacid herbicides.

Experiments were conducted in an attempt to elucidate some of the factors affecting the particle beam mass spectra of the phenoxyacid herbicides. MCPA and 2,4-D were examined with and without ammonium acetate in the mobile phase and at two concentration levels, 0.5 and $2.0 \mu \mathrm{g}$ per injection. These experiments were run without a column using flow injection at a flow rate of $0.4 \mathrm{~mL} / \mathrm{min}$, at an ion source temperature of $250{ }^{\circ} \mathrm{C}$. The relative abundance of ions indicative of particle beam mass spectral variation in MCPA and 2,4-D are listed in Table 1 by experimental parameter. Abundance data were derived from background subtracted mass spectra averaged over a minimum of three data points measured across the top half of the peak. The experiments were conducted on a single day with three injections at each experimental condition for each compound.

The mass spectra utilized in Table 1 are not library matchable, suggesting that at least some of the factors affecting mass spectral quality were in operation. Sta- tistical analysis ( $t$ test, $p=0.05$ ) of the data revealed that the relative abundance of certain variable intensity ions was dependent on the amount of compound injected for both MCPA and 2,4-D with or without ammonium acetate in the mobile phase. In addition, the presence of ammonium acetate in the mobile phase had a significant influence on the relative abundance of the variable intensity ions for 2,4-D at both high and low concentrations and for MCPA at the higher concentration only.

For MCPA, the ion ratio $m / z$ 141:142 exhibited a significant decrease at the lower concentration level as did the relative abundance of the ion at $m / z 141$ and the molecular ion at $m / z$ 200. For 2,4-D the ions at $m / z 128,139$, and 168 all exhibited a significant increase in relative abundance at the lower concentration level. At a given concentration, the presence of ammonium acetate in the mobile phase resulted in a significant decrease in the relative abundance of the ions at $m / z 128,139$, and 168 . These data indicate that at low concentration the particle beam mass spectra of MCPA and 2,4-D can deviate substantially from reference spectra. In addition, the presence of ammonium acetate in the mobile phase suppressed formation of the variable intensity ions for 2,4-D and for MCPA (at high concentration). Ammonium acetate may exert its influence through the "carrier effect" by enhancing nominal analyte concentration in the ion source [20].

Table 2 presents the effect of ion source temperature on mass spectral quality with a clean ion source. The mobile phase was $100 \%$ acetonitrile, and both compounds were introduced into the particle beam system by flow injection $(2 \mu \mathrm{g})$ at a flow rate of $0.4 \mathrm{~mL} / \mathrm{min}$.

Table 1. Effects of concentration and mobile phase on mass spectral quality $(n=3)^{*}$

\begin{tabular}{|c|c|c|c|c|c|c|c|}
\hline \multirow[b]{2}{*}{ Compound } & \multirow{2}{*}{$\begin{array}{l}\text { Amount } \\
\text { (ng) }\end{array}$} & \multirow{2}{*}{$\begin{array}{l}\text { Mobile } \\
\text { phase }\end{array}$} & \multicolumn{5}{|c|}{$\begin{array}{c}\text { lons }(m / z) \\
(\% \text { relative abundance })\end{array}$} \\
\hline & & & 128 & 139 & 162 & 168 & 220 \\
\hline \multirow[t]{7}{*}{$2,4-D$} & 2500 & $\overline{\mathrm{D} \mid \mathrm{P}}$ & 4 & 2 & 100 & 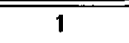 & 50 \\
\hline & & & $s$ & $\bar{x}$ & $s$ & $\bar{x}$ & $s$ \\
\hline & 500 & 1 & $92 \pm 6.9$ & $75 \pm 4.9$ & $100 \pm 0.6$ & $64 \pm \mathbf{3 . 3}$ & $7.0 \pm 3.4$ \\
\hline & 500 & 2 & $67 \pm 7.5$ & $62 \pm 2.6$ & $100 \pm 0.0$ & $51 \pm 2.8$ & $7.6 \pm 0.5$ \\
\hline & 2000 & 1 & $59 \pm 5.2$ & $59 \pm 4.1$ & $100 \pm 0.0$ & $45 \pm 4.3$ & $8.3 \pm 1.8$ \\
\hline & 2000 & 2 & $44 \pm 5.3$ & $47 \pm 4.2$ & $100 \pm 0.0$ & $34 \pm 2.0$ & $9.7 \pm 0.6$ \\
\hline & & & 107 & 141 & 142 & 200 & $141 / 142$ \\
\hline \multirow[t]{6}{*}{ MCPA } & 2500 & DIP & 19 & 100 & 34 & 76 & 2.94 \\
\hline & & & $\bar{x}$ & $s$ & $\bar{x} \quad s$ & $\bar{x}$ & $\bar{x}$ \\
\hline & 500 & 1 & $100 \pm 0.0$ & $43 \pm 9.0$ & $92 \pm 8.0$ & $15 \pm 3.8$ & $0.47 \pm 0.08$ \\
\hline & 500 & 2 & $100 \pm 0.0$ & $46 \pm 2.2$ & $89 \pm 3.2$ & $18 \pm 1.7$ & $0.52 \pm 0.04$ \\
\hline & 2000 & 1 & $100 \pm 0.0$ & $61 \pm 4.8$ & $94 \pm 4.6$ & $24 \pm 0.4$ & $0.65 \pm 0.04$ \\
\hline & 2000 & 2 & $98 \pm 2.1$ & $79 \pm 2.3$ & $97 \pm 4.5$ & $35 \pm 1.5$ & $0.81 \pm 0.02$ \\
\hline
\end{tabular}

* Mobile phase 1: $50 \%$ methanol, 50\% water with $1 \%$ acetic acid.

Mobile phase 2: $50 \%$ methanol, $50 \%$ water containing $1 \%$ acetic acid and $0.1 \mathrm{M}$ ammonium acetate. $\bar{x}=$ mean relative abundance of three measurements.

$s=$ standard deviation of three measurements.

$\mathrm{DIP}=$ direct insertion probe mass spectrum.

$n=$ number of replicate measurements. 
Table 2. Effects of source temperature on mass spectral quality

\begin{tabular}{|c|c|c|c|c|c|c|}
\hline \multirow[b]{2}{*}{ Compound } & \multirow[b]{2}{*}{ Temperature $\left({ }^{\circ} \mathrm{C}\right)$} & \multicolumn{5}{|c|}{$\begin{array}{c}\text { lons }(m / z) \\
\text { (\% relative abundance) }\end{array}$} \\
\hline & & 128 & 139 & 162 & 168 & 220 \\
\hline \multirow[t]{5}{*}{$\overline{2.4-D}$} & 200 & 22 & 28 & 100 & 18 & 18.8 \\
\hline & 225 & 22 & 21 & 100 & 14 & 14.0 \\
\hline & 250 & 37 & 28 & 100 & 17 & 14.5 \\
\hline & 300 & 43 & 32 & 100 & 18 & 15.3 \\
\hline & & 107 & 141 & 142 & 200 & \\
\hline \multirow[t]{4}{*}{ MCPA } & 200 & 47 & 100 & 54 & 47.8 & \\
\hline & 225 & 90 & 100 & 85 & 39.3 & \\
\hline & 250 & 90 & 100 & 86 & 40.1 & \\
\hline & 300 & 100 & 77 & 87 & 31.6 & \\
\hline
\end{tabular}

For MCPA at $200{ }^{\circ} \mathrm{C}$, ion abundances were observed that were library matchable. Upon heating the source to $300{ }^{\circ} \mathrm{C}$, the ion at $m / z 107$ became the base peak and $m / z 142$ became larger than $m / z$ 141. Similar behavior was observed for 2,4-D, where the "variable intensity" ion at $m / z 128$ increased in relative abundance with increasing temperature. Although the spectra at $200{ }^{\circ} \mathrm{C}$ are of better quality for library matching, the corresponding total iun chrumatugram peaks exhibited extensive tailing at the lower temperatures. The peak shape of the acids became sharper upon heating towards $300{ }^{\circ} \mathrm{C}$, but spectral quality suffered as seen in Table 2 .

Two other factors briefly examined were column bleed and water quality. Although coltumn bleed could have a long-term effect on the cleanliness of the ion source, the problem of spectral quality persisted during flow injection without an analytical column. In addition, the use of water from an in-house water purification system reduced the relative abundance of the ions $m / z 107$ and 142 from MCPA and $m / z$ 128, 139 , and 168 from 2,4-D compared with the use of commercially available HPLC grade water. We found some commercial grade HPLC water left residue behind after filtration or evaporation. This possibly could affect source cleanliness.

The results described to this point indicate that the particle beam mass spectra of phenoxyacid herbicides are variable and can depend on ion source cleanliness, source temperature, and analyte concentration. We postulate that the variation observed in these spectra is due to competing thermal decomposition mechanisms and that the extent of thermal decomposition is variable. Upon entering the ion source, a fraction of the phenoxyacid particles are instantaneously vaporized on impact with ion source surfaces, are ionized, and subsequently undergo expected gas-phase fragmentation. However, another fraction of the phenoxyacid particles are strongly adsorbed onto ion source surfaces where thermal decomposition occurs. The products of thermal decomposition then desorb and are ionized to give rise to characteristic ions in the mass spectra. For MCPA, "thermal decomposition" ions are $m / z 107$ and 142. For 2,4-D, the "thermal decomposition" ions are $m / z 128,139,168$, and, to some extent $m / z 162$.

The changes in the ion ratio between $m / z 141$ and $m / z 142$ for MCPA were further investigated. The DIP mass spectrum of MCPA shows the ratio between $\mathrm{m} / \mathrm{z}$ 141 and $m / z 142$ to be about 3:1. The particle beam LC/MS used in the current work will produce a ratio of 3:1 only with a scrupulously clean ion source and source temperature of $200{ }^{\circ} \mathrm{C}$. After a short period of operation under "normal" conditions, the ion at $m / z$ 142 increased in abundance and overtook $m / z 141$. Figure 3 displays the normalized ion chromatograms of $m / z 107,141,142$, and 200 from MCPA at an ion source temperature of $200{ }^{\circ} \mathrm{C}$. Note that $\mathrm{m} / z 142$ and 107 track each other, but deviate considerably from the $m / z 200$ and 141 profiles. This figure indicates that the species responsible for the ion at $m / z 142$ is residing in the ion source for a longer period of time than the species giving rise to the ion at $m / z 141$. Normalized ion chromatograms have been used to explain surfaceassisted ionization mechanisms [21]. The normalized ion chromatograms suggest that the ion at $\mathrm{m} / \mathrm{z} 142$ might be formed after the molecular species has been altered by adsorption on the source surfaces. This

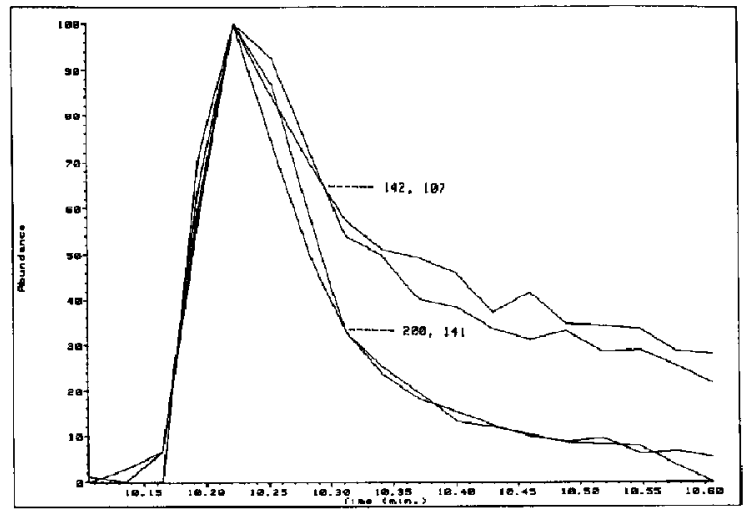

Figure 3. Normalized ion chromatograms from the particle beam mass spectrum of MCPA. 
altered species could then desorb from the source surface and ionize with the formation of $m / z$ 142. A possible candidate for the altered species in this case is the corresponding phenol, 2-methyl-4-chlorophenol, which under EI generates the molecular ion at $m / z$ 142 (base peak) and also an intense ion at $m / z$ 107. It is these two ions, the $m / z 142$ and 107, that exhibit the most variation in abundance in the particle beam mass spectra of MCPA.

Normalized ion chromatograms were generated for several of the ions in the particle beam mass spectrum of 2,4-D and are displayed in Figure 4. This figure indicates that the species responsible for $m / z 128$ and 139 remain in contact with the ion source surfaces for a longer period of time than the molecular species. The $m / z 161$ ion profile tracks that of the molecular ion, $m / z 220$, indicating that it is probably formed directly from 2,4-D and not from a thermal decomposition product. The $m / z 162$ ion from 2,4-D also shows a wider profile than the molecular ion at $m / z 220$, but not as broad as the $m / z 128$ and 139 ions. There is a possibility that the $m / z \quad 162$ is coming from two sourres: a fragment from EI of 2,4-D and the molecular ion from the thermal decomposition product, 2,4-dichlorophenol.

Electron ionization of phenoxyacids is thought to produce, in addition to ions corresponding to fragmentation of the substituted aromatic ring, ions corresponding to loss of carboxylate $[\mathrm{M}-\mathrm{COOH}]^{+}$, loss of the carboxylic acid $[\mathrm{M}-\mathrm{RCOOH}]^{+}$, loss of the carboxylic acid ether $[\mathrm{M}-\mathrm{ORCOOH}]^{+}$, and loss of the carboxylic acid with hydrogen migration [M $\mathrm{RCOO}^{+}$[22]. The last ion described is frequently the base peak in phenoxyacid mass spectra and is thought to arise from transfer of the acid proton to the phenolic oxygen. This mechanism may account for the ions $m / z$ 142 from MCPA and $m / z 162$ from 2,4-D. If this mechanism were operating, the ion chromatograms for these ions would be expected to track the ion chromatograms for the corresponding molecular ions. As shown in Figures 3 and 4, they do not.

Normalized ion chromatograms were also used to

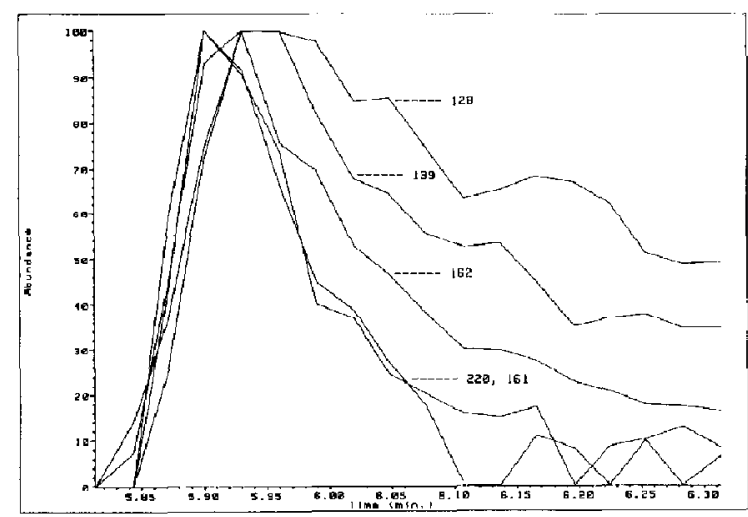

Figure 4. Normalized ion chromatograms from the particle beam mass spectrum of 2,4-D. examine the particle beam positive chemical ionization (PCI) mass spectra of MCPA and 2,4-D. The spectra were acquired using flow injection (2 $\mu \mathrm{g}$ ) in $100 \%$ methanol, methane reagent gas, and an ion source temperature of $250^{\circ} \mathrm{C}$. The PCI spectrum of MCPA is shown in Figure 5 . The extensive fragmentation (or apparent fragmentation) and the lack of an abundant pseudomolecular ion $[\mathrm{M}+\mathrm{H}]^{+}$at $m / z 201$ was somewhat unexpected. The ions at $m / z 183$ and 155 can be explained by straightforward gas-phase fragmentation, namely, loss of the neutral molecules $\mathrm{HOH}$ and $\mathrm{HCOOH}$, respectively, from the pseudomolecular ion. The ion at $m / z 143$ is difficult to rationalize in terms of neutral molecule loss from the pseudomolecular ion. We suspect that a major portion of the ion at $m / z 143$ is the pseudomolecular ion of a MCPA thermal decomposition product, 2-methyl-4-chlorophenol. This notion is supported by the normalized ion chromatograms shown in Figure 6. The expected fragment ion at $\mathrm{m} / \mathrm{z}$ 155 tracks the MCPA pseudomolecular ion but the ion at $m / z 143$ does not.

Similarly, the PCI spectrum of 2,4-D exhibited extensive apparent fragmentation with little pseudomolecular ion. Expected 2,4-D fragment ions resulting from the loss of $\mathrm{HOH}$ and $\mathrm{HCOOH}$ were present at $m / z 203(8 \%)$ and $m / z 175(36 \%)$. The most intense ions, $m / z 129$ (base peak) and $m / z 163(71 \%$ ) are difficult to rationalize in terms of neutral molecule loss. These ions may be pseudomolecular ions of $2 x_{r}^{4-D}$ thermal decomposition products. The normalized ion chromatograms of the ions at $m / z 129,163$, and 175 show $m / z 129$ and 163 to track each other in a much broader trace than that for $m / z 175$.

At an ion source temperature of $150^{\circ} \mathrm{C}$, MCPA and 2,4-D still exhibited extensive apparent fragmentation under PCI conditions, although the relative abundance of the pseudomolecular ion did increase. For MCPA, the relative abundance of $m / z 201$ increased (36\%), the $m / z 143$ decreased (25\%), and the $m / z 155$ remained the base peak. For 2,4-D, the relative abundance of $m / z 221$ increased (36\%), the $m / z 129(11 \%)$

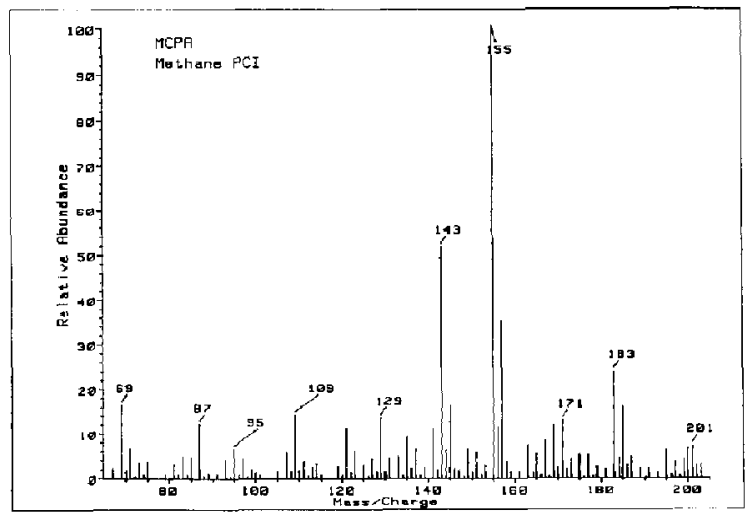

Figure 5. Particle beam positive chemical ionization mass spectrum of MCPA. 


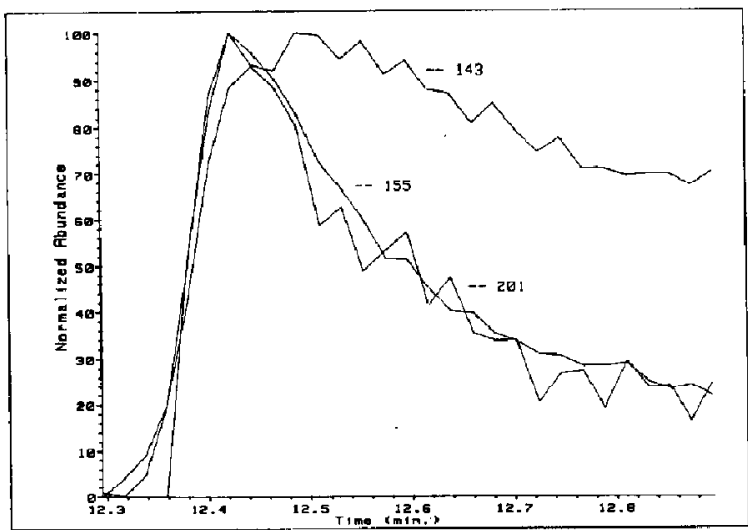

Figure 6. Normalized ion chromatograms from the particle beam positive chemical ionization mass spectrum of MCPA.

and $m / z 163(15 \%)$ decreased substantially, and $m / z$ 175 became the base peak. The results at lower ion source temperature are consistent with the concept of thermal decomposition contributions to the PCI spectra of MCPA and 2,4-D. Both fragmentation and thermal decomposition decreased at lower temperatures.

To understand more about the structures of the thermal decomposition ions, tandem mass spectrometry experiments were performed on a triple quadrupole mass spectrometer. The GC was used to introduce the corresponding phenol into the triple quadrupole mass spectrometer. Figure 7a shows the daughter ion CAD experiments from the molecular ion of 2,4-dichlorophenol $(m / z 162)$. It is a simple spectrum; the chlorine isotopes have been removed because the spectrum was the CAD experiment on an ion with only a ${ }^{35} \mathrm{Cl}$ contribution. The spectrum shows the same major ions present in the EI spectrum of this compound.

The next experiment used the ThermaBeam LC/MS interface connected to a triple quadrupole mass spectrometer. Figure $7 \mathrm{~b}$ shows the CAD daughter ion spectrum of $m / z 162$ from 2,4-D. This spectrum is almost identical to that shown in Figure 7a from 2,4-dichlorophenol. The CAD daughter ion spectrum of $m / z 128$ from 2,4-D is shown in Figure 7c. This spectrum is similar to the EI spectrum for monochlorophenol [23].

Similar behavior is shown by MCPA. These experiments indicate that the $m / z 142$ ion from MCPA is similar to the molecular ion from the corresponding free phenol. This similarity is not sufficient to distinguish the origin of $m / z 142$ as an EI fragment of the MCPA molecular ion or the molecular ion of 4-chloro2-methylphenol resulting from thermal decomposition of MCPA, because the postulated hydrogen migration mechanism leading to $[\mathrm{M}-\mathrm{RCOO}]^{+}$predicts a structure identical to the molecular ion of the corresponding phenol [22]. However, the CAD parent ion spectrum of $m / z 141$ exhibited a $m / z 200$ ion but the parent ion spectrum of $m / z 142$ did not exhibit any apparent precursor ion (Figure $8 \mathrm{a}$ and $\mathrm{b}$ ). These experiments suggest the possibility that $m / z 142$ is itself a
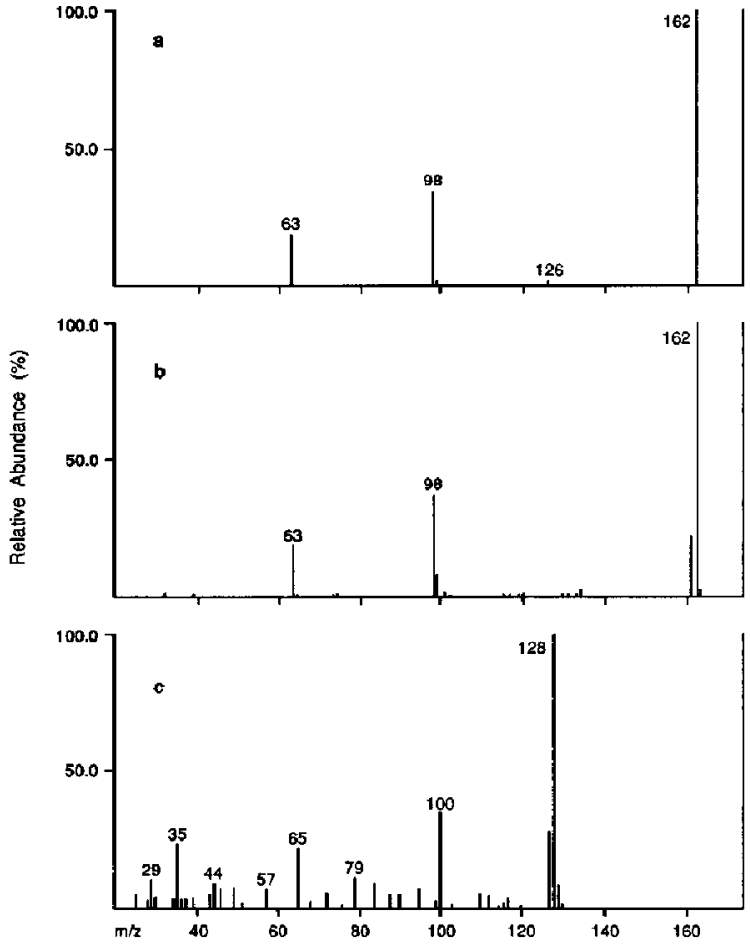

Figure 7. (a) Daughter ion CAD spectrum of $m / z 162$ from 2,A-dichlorophenol. (b) Daughter ion CAD spectrum of $m / z 162$ from 2,4-D. (c) Daughter ion CAD spectrum of $m / z 128$ from 2,4-D.

molecular ion species and does not arise directly from fragmentation of the MCPA molecular ion. We propose that $m / z 142$ is formed for the most part by adsorption of MCPA onto ion source surfaces, where it thermally decomposes to the corresponding phenol, is desorbed, and then ionized in the vapor phase. The $m / z 107$ ion may be accounted for by fragmentation of the $m / z 142$ ion. While the lack of an $m / z 200$ ion in the parent ion spectrum of $m / z 142$ does not constitute proof, the experiment is relevant because an intense $m / z 200$ signal would refute the argument that $m / z 142$ does not arise from $m / z 200$.

The parent ion spectrum of $m / z 162$ from 2,4-D exhibited a very small $m / z 220$ ion contribution, which is consistent with the supposition that the $m / z 162$ ion is coming partly from the EI of 2,4-D and is also the molecular ion of 2,4-dichlorophenol. The parent ion spectrum of $\mathrm{m} / \mathrm{z} 128$ from 2,4-D exhibited no apparent precursor ion. Thus, the $m / z 128$ ion could be the molecular ion from chlorophenol formed from the thermal decomposition of 2,4-D. In addition, the CAD parent ion spectrum of $m / z 139$ exhibited an ion at $m / z 168$ with no other higher mass precursor ions apparent. We conclude that (1) 2,4-D undergoes thermal decomposition to some extent and the $m / z 128$ and 139 are evidence of thermal decomposition, (2) the $m / z 162$ arises from both EI and thermal decomposition mechanisms, and (3) other ions such as those 


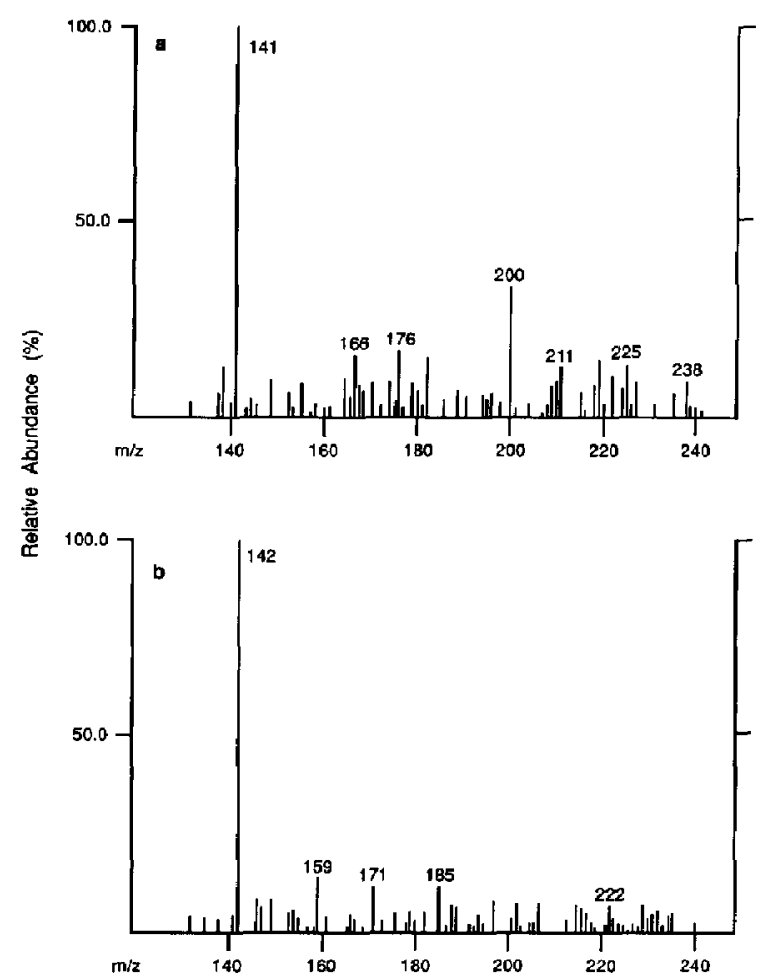

Figure 8. (a) Parent ion CAD spectrum of $m / z 141$ from MCPA. (b) Parent ion CAD spectrum of $m / z 142$ from MCPA.

at $m / z 175$ and 161 arise directly from 2,4-D by EI mechanisms.

\section{Summary}

There appears to be substantial interaction of the phe noxyacid herbicides with the ion source surface. This interaction may lead to thermal decomposition of the analytes and give rise to spectral variation. The particle beam EI mass spectra of phenoxyacids are possibly composite spectra with contributions from EI and thermal decomposition mechanisms. Variation in mass spectral quality depends on ion source cleanliness, source temperature, and analyte concentration. There may be other parameters that might contribute to spectral variation that have not been identified here. One group of researchers has generated spectra by $\mathrm{PB}$ LC/MS for these herbicides without much evidence of thermal decomposition [16], although the molecular ion cluster is less intense than in reference EI libraries. In this present work, the use of two different PB LC/MS systerns has shown the possibility of thermal decomposition. Other laboratories have shown similar results [19]. The chlorinated phenoxyacid herbicides may represent an extreme case in spectral variation. In this respect, they may be useful as diagnostic compounds to monitor the performance of $\mathrm{PB}$ systems. For example, if the $m / z 128$ ion from $2,4-\mathrm{D}$ becomes the base peak, the ion source needs cleaning. As long as volatilization takes place on the walls of the ion source, thermal decomposition for compounds such as these herbicides remains a real possibility.

\section{Notice}

Although the research described in this article has been funded wholly by the U.S. Environmental Protection Agency through contract number 68-CO-0049 to Lockheed Engineering and Sciences Company, it has not been subjected to Agency review. Therefore, it does not necessarily reflect the views of the Agency. Mention of trade names or commercial products does not constitute endorsement or recommendation for use.

\section{References}

1. Willoughby, R. C.; Browner, R. F. Anal. Chem. 1984, 6, 2626.

2. Gurka, D. F.; Shore, F. L.; Pan, S. T.; Amick, E. N. I. Assoc. Off. Anal. Chem. 1986, 69, 970.

3. Gurka, D. F.; Shore, F. L.; Pan, S. T. I. Assoc. Off. Anal. Chem. 1987, 70, 889 .

4. Test Methods for Evaluating Solid Waste, 3rd ed.; SW-846; U.S. Environmental Protection Agency, Washington, DC, 1986.

5. Lopez-Avila, V.; Hirata, P.; Kraska, S.; Taylor, J. H. I. Agric. Food Chem. 1986, 34, 530.

6. Voyksner, R. D.; Bursey, J. T.; Pellizzari, E. D. T. Chromatogr. 1984, 312, 221.

7. Geerdink, R. B; Maris, F. A.; DeJong, G. I.; Frei, R. W.; Brinkman, U. A. Th. J. Chromatogr. 1987, 394, 51.

8. Voyksner, R. D. In Applications of New Mass Spectrometry Techniques in Pesticide Chemistry; Rosen, J. D., Ed.; Wiley: New York, 1987.

9. Barcelo, D. Chromatographia. 1988, 25, 295.

10. Barcelo, D. Org. Mass Spectrom. 1989, 24, 219.

11. Barcelo, D. Org. Mass Spectrom. 1989, 24, 898.

12. Jones, T. L.; Betowski, L. D.; Yinon, J. In Liquid Chromatography/Mass Spectrometry: Applications in Agricultural, Pharmaceutical, and Environmental Chemistry; Brown, M., Ed; ACS Symposium Series, No. 420; Washington, DC, 1990; p. 62.

13. Budde, W.; Behymer, T. J. Am. Water Works Assac. 1990, 82, 60.

14. Brown, M. A.; Kim, I. S.; Sasinos, F. I.; Stephens, R. D. In Liquid Chromatography/Mass Spectrometry: Applications in Agricultural, Pharmaceutical, and Environmental Chemistry; Brown, M., Ed.; ACS Symposium Series, No. 420; Washington, DC, 1990; p. 198.

15. Mattina, M. J. I. J. Chromatogr. 1991, 542, 385.

16. Kim, I. S.; Sasinos, F. I.; Stephens, R. D.; Wang, J.; Brown, M. A. Anal. Chem. 1991, 63, 819.

17. McLafferty, F.; Stauffer, D. The Wiley Library/NBS Registry of Mass Spectral Data; Wiley: New York, 1988.

18. CRC Handbook of Mass Spectra of Environmental Contaminants; Hites, R. A., Ed.; CRC Press, Boca Raton, FL, 1985.

19. Jones, T. L.; Betowski, L. D.; Lesnik, B.; Chiang, T. C.; Teberg, J. E. Environ. Sci. Tech. 1991, 25, 1880.

20. Bellar, T. A.; Behymer, T. D.; Budde, W. L. I. Am. Soc. Mass Spcctrom. 1990, 1, 92.

21. Sears, L. J.; Champbell, J. A.; Grimsrud, E. P. Biomed. Environ. Mass. Spectrom. 1987, 14, 401.

22. Nolte, J.; Paschold, B.; Kettrup, A. Int. J. Enoiron. Anal. Chem. 1983, 15, 231.

23. Heller, S. R,; Milne, G. W. A. EPA/NIH Mass Spectral Data Base 1978, NSRDS-NBS 63, U.S. National Bureau of Standards, p. 142. 